\title{
Research on Interactive Scenarios and Mechanisms of Energy Supply and Use Modules of Park-level Integrated Energy System
}

\author{
Zhao Pengxiang ${ }^{1}$, Li Na $^{1}$, Li Zhiyuan ${ }^{1 *}$, Wang Nan $^{1}$ \\ ${ }^{1}$ State Grid Integrated Energy Service Co., Ltd.100052, Beijing, China
}

\begin{abstract}
The integrated energy system can realize the complementary advantages of multiple energy sources and effectively solve the problem of low integrated energy efficiency of traditional energy systems. Carrying out research on the interactive scenarios and mechanisms of the energy supply and use modules of the integrated energy system is conducive to promoting the development of clean energy and promoting the indepth integration of energy Internet and distributed energy technology, smart grid technology, and energy storage technology. First, it summarizes the scenarios of the energy supply and use modules of the integrated energy system, and analyzes the interaction mechanism and paths of the energy supply and use modules of the integrated energy system on this basis.
\end{abstract}

\section{Introduction}

In order to promote the consumption of renewable energy and fully explore the potential of various energy systems such as power supply, heating, and gas supply, an integrated energy system is derived from the traditional distributed energy system. It integrates electricity, heat, gas and other energy forms to produce and supply energy on demand. It not only realizes the complementary advantages of multiple energy sources, but also effectively solves the problem of low comprehensive energy efficiency of traditional energy systems. However, due to the failure of energy supply modules or energy transportation pipelines in the integrated energy system, and the dynamic changes of the loads on both sides of the supply and demand due to a large number of uncertain factors, in order to ensure the reliability, economy and environmental protection of the system, there are It is necessary to study the interactive scenarios and mechanisms of the integrated energy system, so that the electric cooling and heating load can be synchronized and optimized, and the flexibility of the integrated energy system should be improved.

Literature[1-3] uses electricity-to-gas and electricityto-heat technology to promote the consumption of renewable energy, and at the same time it plays the role of peak-shaving and valley-filling. It is an important means to improve the comprehensive energy efficiency of the integrated energy system. Literature [4-5] uses the scene analysis method to describe the uncertainty of wind power, and proposes to use the importance sampling method to analyze the scene. Compared with the traditional scene generation method, this method does not reduce the accuracy of the calculation cost. Literature [6] considers the application of wind power hydrogen production equipment in the integrated energy system, takes into account the mutual conversion between multiple energy sources, and analyzes the importance of wind power hydrogen production equipment for reducing system operating costs and increasing clean energy consumption. effect. Literature [7-9] considers the dispatchability of various loads on the demand side, comprehensively considers the complementary advantages between the power system and the thermal system, and saves the energy consumption cost of the integrated energy system, but it does not consider users when establishing the thermal load demand response subjective will. Literature [10] takes the supply-demand interaction of cold, heat, and electricity as its foothold, based on the master-slave game theory, uses the price of cold, heat, and electricity multiple energy sources and the hourly load of users as decision variables. An interactive game was launched in the energy transaction in 2012, and a reasonable equilibrium solution of the master-slave game was obtained. Literature [11-14] summarizes the topological characteristics of the regional integrated energy system, and establishes a topological description model of the energy system based on the energy flow balance constraints and the characteristics of the heating network. Literature [15-18] established a thermal system energy flow model compatible with the power system model, taking into account the constraints of the heat exchange link, and making the optimal dispatch results of the integrated energy system more accurate and credible.

Most of the research on the optimization of integrated 
energy systems focuses on a single scenario to formulate integrated energy system operation control strategies, but few articles summarize and organize multiple interactive scenarios of integrated energy systems. Combining the above background, this article takes the energy supply and consumption scenarios of the park-level integrated energy system as the research object, and studies the interaction scenarios and mechanisms of the energy supply and use modules.

\section{The interactive scenario of the energy supply and use modules of the integrated energy system}

\subsection{The economic improvement scenario}

The composition of the integrated energy system source, network, and load-storage interaction between the participating entities under the scenario of improving their own economic efficiency is shown below.

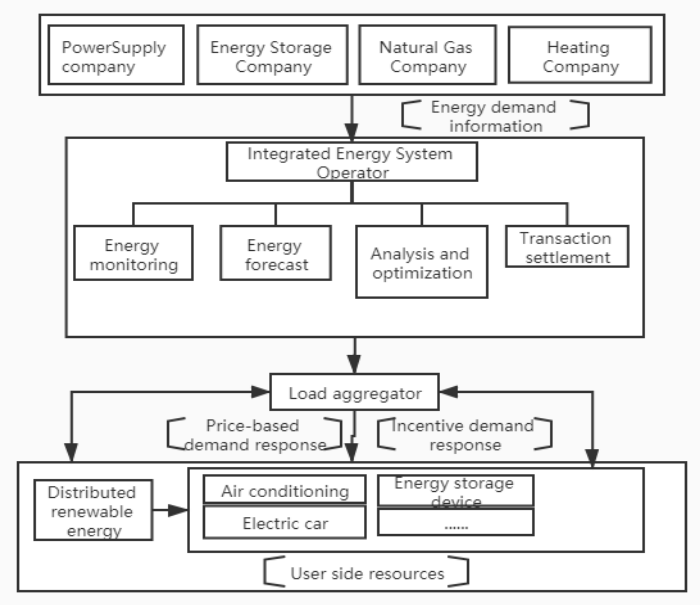

Figure 1. The source-network-load-storage interaction of the integrated energy system under the scenario of improving its own economy

(1) Energy factors. The energy categories in this scenario mainly focus on renewable energy such as wind and light on the primary side, as well as electricity and heat on the demand side.

(2) Main elements. The participants in this scenario seek to improve their own economy. Participants include system operators, load aggregators, power generation companies, natural gas companies, thermal power companies, energy storage companies, and users. The integrated energy system operator is mainly responsible for forecasting and monitoring the supply and demand of energy, and conveying energy price information to the load aggregator; the main responsibility of the load aggregator is to gather the responsive demand-side resources of demand-side users .Taking the aggregator as an individual to participate in the dispatch of the integrated energy system, and automatically control the user's comprehensive demand response actions; the main responsibility of power generation companies, energy storage companies, natural gas companies, heating companies and other energy suppliers is to feed back the real-time price of energy to the system Operators: The main responsibility of users is to interconnect their responsive resources with the aggregator's platform through the network, observe the response, adjust or change the response plan formulated by the load aggregator, and suspend the response process.

(3) The target element implements an interactive and coordinated arrangement of the operation strategy of the source, network, load, and storage links through the source, network, load and storage, and makes full use of the characteristics of various resources to jointly promote the optimized operation of the system in a flexible and efficient manner, and promote the efficient consumption of clean energy. Increase energy supplier profits, while energy users can respond to price signals in multiple energy markets, and adjust their own different types of energy use needs and habits according to price signals, thereby reducing their own energy costs, distributed The access of energy storage, heat storage, and electric vehicles increases the flexibility of users' energy use, enabling users to have larger capacity "virtual energy units" that can directly participate in ancillary services or energy market transactions and increase their own profits.

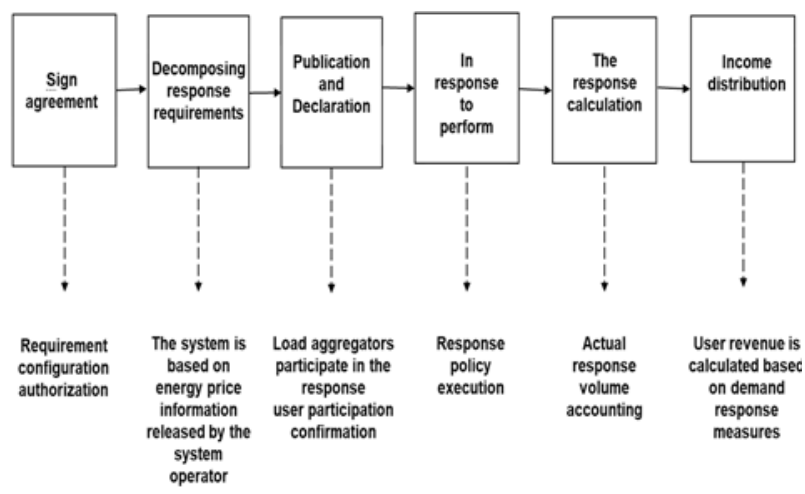

Figure 2. The source-network-load-storage interaction of the integrated energy system under the scenario of improving its own economy

\subsection{Promoting the Distributed Renewable Energy Consumption Scenario}

The following shows the integrated energy system sourcenetwork-load-storage interaction architecture diagram between the participating entities in the distributed renewable energy consumption scenario. 


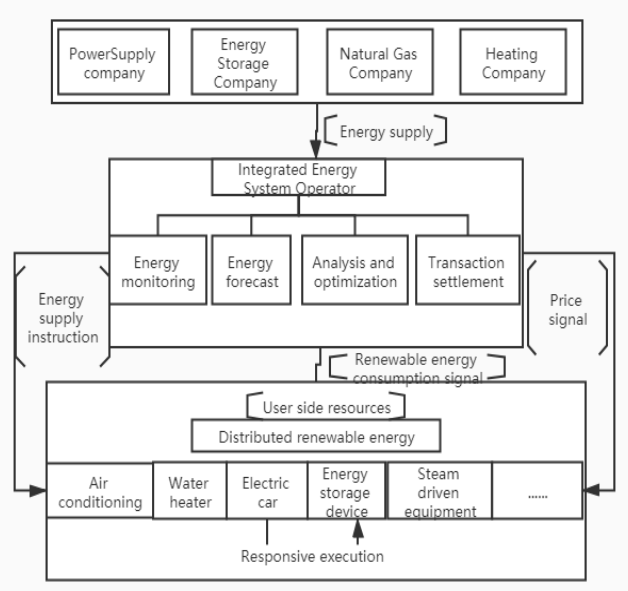

Figure 3. Promoting the interaction of source, network, load and storage in the integrated energy system under

the scenario of distributed renewable energy consumption

(1) Energy factors. The energy categories in this scenario include renewable energy such as primary wind energy and solar energy, as well as demand-side electricity, heat, and cold.

(2) Main elements. The main requirement in this scenario is to complete distributed renewable energy consumption. Participants include system operators, distributed renewable energy, power generation companies, energy storage companies, natural gas companies, thermal power companies, and users.

(3) Target elements. With the massive access of intermittent and fluctuating distributed renewable energy sources, the lack of system regulation capabilities and the slowdown in energy demand have led to the dilemma of renewable energy consumption.

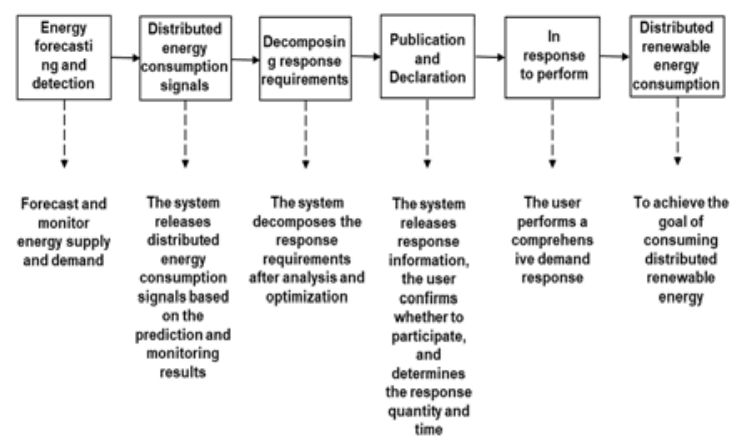

Figure 4. Promoting the interaction between source network, load and storage of integrated energy system under the scenario of Distributed Renewable Energy Consumption of the integrated energy system's source-network loadstorage interaction from two aspects: the realization mechanism and realization path of the integrated energy system's source-network load-storage interaction.

\subsection{Interaction realization mechanism}

Specifically, its interaction realization mechanism mainly includes the following two aspects.

1) Horizontal multi-source complementary. "Horizontal multi-source complementarity" is derived from the concept of "source-source complementation" in the power system. "Horizontal multi-source complementation" in the integrated energy system refers to multiple energy sources such as power systems, petroleum systems, heating systems, and natural gas supply systems. Complementary coordination between resources, highlighting the "substitutability" between various types of energy, users can not only choose

different energy sources, but also freely choose the way of obtaining energy resources.

2) Coordination of vertical source network and load storage. The "vertical source-network-load-storage coordination" in the integrated energy system mainly refers to two aspects: (1)The development and utilization of energy resources, the resource transportation network, and the energy transmission network are realized through a variety of energy conversion technologies and information flow and energy flow interaction technologies Coordination between users; (2)Unify the user 's multiple energy demand into a whole, further expand the power demand side management into "comprehensive energy management" in the entire energy field, and use the broad demand side resources to promote clean energy consumption. The role of receiving and ensuring the safe and stable operation of the system has been further amplified.

The main structure of the interaction mechanism between the source network and the load storage of the integrated energy system is shown in Figure 5.

\section{The interaction mechanism and path of the energy supply and use modules of the integrated energy system}

This section will study the interaction mechanism and path 


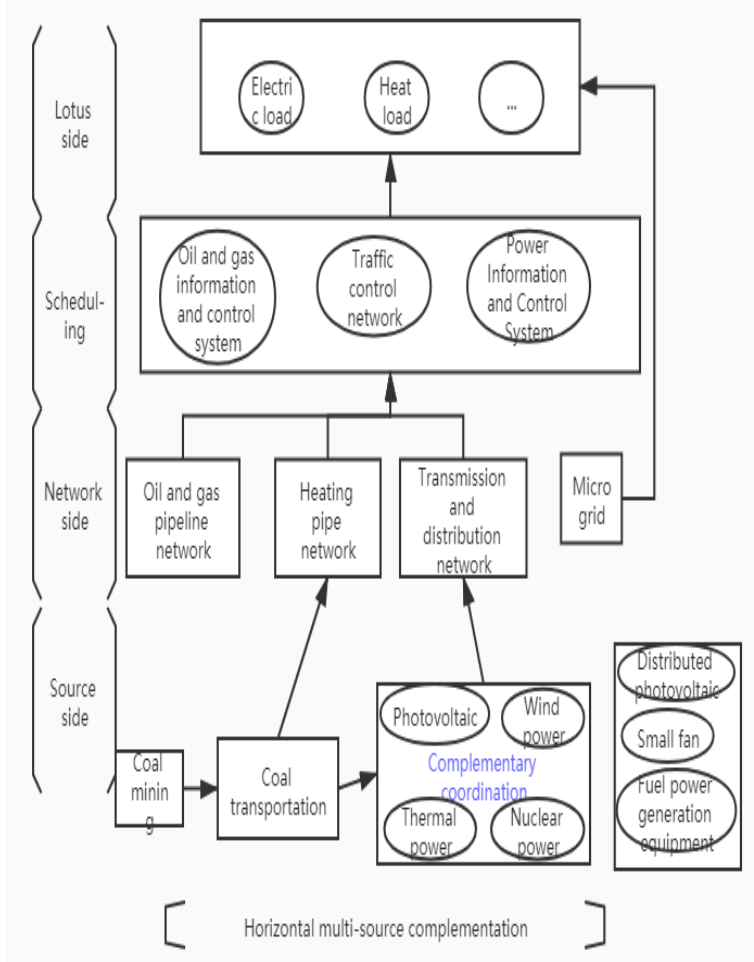

Figure5. Architecture diagram of the interaction mechanism between the source network and the load storage of the integrated energy system

\subsection{Interactive realization path}

The realization path of the source-network-load-storage interaction of the integrated energy system is mainly divided into 4 parts, as shown in Figure 3-2 in chronological order.

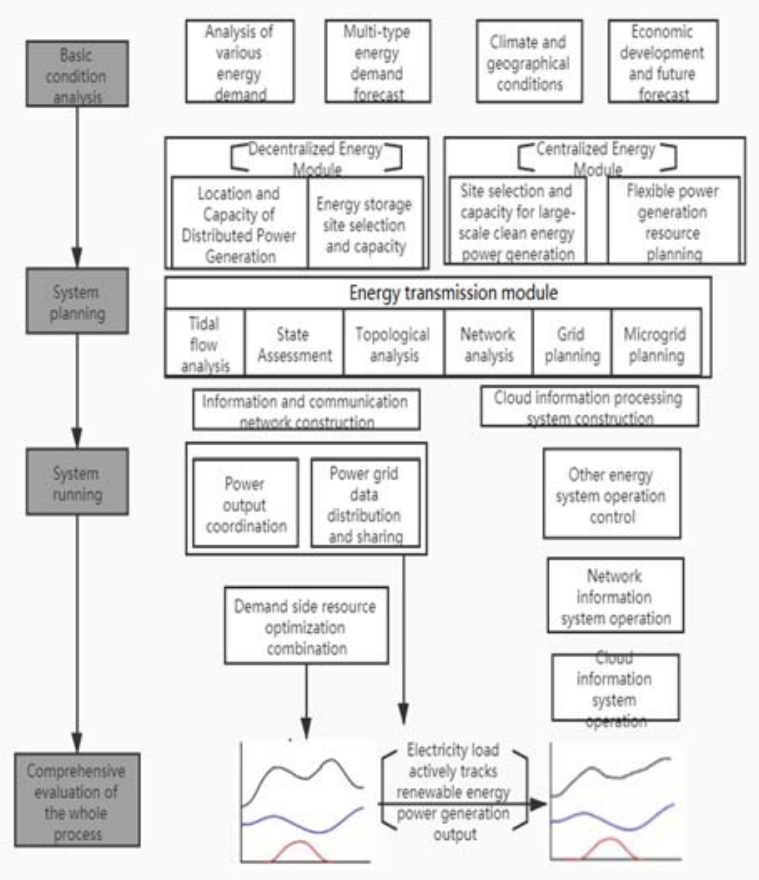

Figure 6.The basic flow of the integrated energy system's source-network-load-storage interaction realization path
Here, from the perspective of an integrated energy system investment operator, the realization path of the interaction between the source, network and load of the entire integrated energy system is explained. The details are as follows:

(1) Analysis of basic conditions. Before the system planning scheme design, the system operator needs to evaluate the current economic situation of the construction target area and forecast the future development trend.

(2) System planning. In the planning stage, it is necessary to fully consider the needs of future system interaction operations. The decentralized energy module should provide an effective supplement to the centralized energy module based on the internal self-balance of energy supply and demand; on the other hand, the planning of the centralized energy module must be for operation. The complementary coordination of clean energy and flexible power generation resources at the stage provides the basis.

(3) System operation. In the system operation stage, the system operator collects all user energy consumption information and basic information on the energy supply side through the information communication network.

(4) Whole process evaluation. In the 3-5 years after the integrated energy system is completed and operated, evaluate and analyze the operation of the project, construct a multi-faceted indicator system including renewable energy efficiency, power supply reliability, and equipment utilization, and use comprehensive evaluation methods for comparison.Analyze, find project defects and make cyclic corrections.

From the perspective of the main content of the realization path of the source-network-load-storage interaction of the integrated energy system, this optimization model can coordinate the energy development, energy transmission, energy demand and use of the integrated energy system's source-networkload-storage interaction into one Organic as a whole,such coordinated optimization can not only proceed from a higher level to realize the optimal allocation of energy resources, but also promote the efficient development and utilization of clean energy and increase the proportion of clean energy in the final energy consumption.

\section{Conclusion}

Through the interaction of source, network, load and storage, the safety, flexibility and economy of the integrated energy system can be effectively improved; it can bring economic benefits to various types of entities such as power grid companies, integrated energy service providers, and users; and it can also promote new energy. The consumption of energy has brought significant environmental benefits. In summary, the research on the interactive scenarios and mechanisms of the energy supply and use modules of the integrated energy system will support the reconstruction of the energy production paradigm in the energy production and consumption revolution and the innovative application of localized integrated energy system exploration, enriching the construction of integrated energy systems, energy The 
research coverage of issues such as the realization of the Internet will further promote the development of clean energy and support the efficient use of energy and the construction of energy saving and emission reduction.

\section{References}

1. Yu Xuefeng, Xu Guizhi, LIU Qihui, et al. Modeling and Optimization Operation of integrated energy System of the Park including electric conversion gas and electric conversion heat $[\mathrm{J}]$.Power demand Side Management, 2020, 22(1): 58-63, 80.

2. Wang Xiaoxia, Zhao Lihua, Zou Pinhua. Study on topology Reconstruction method of Multi-heat Source Annular heat Network Fault Conditions [J].Hvac, 2014, 44(9): 85-90.

3. Yu Xuefeng, Xu Guizhi, LIU Qihui, et al. Modeling and Optimization Operation of integrated energy System of the Park including electric conversion gas and electric conversion heat $[\mathrm{J}]$.Power demand Side Management, 2020, 22(1): 58-63, 80.

4. Wang Xiaoxia, Zhao Lihua, Zou Pinhua. Study on topology Reconstruction method of Multi-heat Source Annular heat Network Fault Conditions [J].Hvac, 2014, 44(9): 85-90.

5. Chen Xuefeng. Research on Distribution Network Analysis and Network Reconstruction [D]. [Publication location unknown]: Shenyang University of Technology, 2011.

6. Xia Peng. Research on Multi-objective Distribution Network Reconstruction with Distributed Power Supply [D]. [Publication location unknown]: Xi 'an University of Petroleum, 2019.

7. Zhang Jianghua, Huang Yunfeng, Xi Peifeng. Research on Operation Optimization of regional Integrated Energy System including Wind Power [J].Henan Science and Technology, 2019, 697(35): 148-150.

8. Guo Mengjie, Yan Zheng, Zhou Yun, et al. Optimization operation of integrated energy System for hydrogen production Unit with wind Power [J].China Electric Power, 2020, 53(1): 115-123, 161.

9. Wu Shengcong, Wu Feng, Chen Yuxuan, et al. Research on multi-scene Distribution Network Reconstruction under mixed trend $[\mathrm{J}]$.Automated applications, 2019, Volume deletion (8): 77-78.

10. Fang Shaofeng, Zhou Renjun, Xu Fulu, et al. Optimization operation of integrated energy system of micro-grid in the Park considering comprehensive demand response of various electric heating loads [J].Journal of Power Systems and Automation, 2020, 32(1): 50-57.

11. Xu Yutao, Tan Zhukui, Yuan Xufeng, et al. Distribution Network Reconstruction of distributed Power Supply Access [J].Electric Measurement and Instrumentation, 2019, 56(7): 89-94.

12. Ren Hongbo, WANG Guangtao, LI Qifen, et al. Research on Collaborative Optimization of Equipment Configuration and Operation Strategy of regional integrated Energy System from the perspective of supply-Demand interaction [J].Thermal power generation, 2020, 49(3): 60-67.

13. Wang Xiaoxia, Zou Pinghua, LI Xiangli. Valve Closing Scheme of Heat supply Network Failure [J]. Gas and heat, 2004, volume loss (8): 432-434.

14. Liu Mengjun, Zou Pinghua, TANG Haoxuan, et al. Research on gis-based Spatial Heat Grid Shut-off Valve Scheme [J].Journal of Wuhan University of Technology, 2006, volume missing (1): 51-54.

15. Xu Chengsi, Dong Shufeng, WU Jincheng, et al. Planning of energy stations and pipelines considering topological characteristics of regional integrated energy systems [J].Automation of Electric Power Systems, 2020, 44(3): 74-86.

16. Chen Qun, HAO Junhong, Chen Lei, et al. Integrated Energy Transport Model in electrothermal integrated energy System [J].Power System Automation, 2017, 41(13): 7-13, 69.

17. Xue Yuntao, Chen Yichao, Li Xiuwen, et al. Combined peak-Valley time-sharing pricing Model based on user satisfaction and Ramsey Pricing Theory [J].Power System Protection and Control, 2018, 46(5): 122-128.

18. Yang Shuai, Chen Lei, XU Fei, et al. Optimal dispatching model of wind abandon and absorption of electrothermal integrated energy System based on energy flow [J].Power Grid Technology, 2018, 42(2): 417-426. 